\title{
Perspectives of Pulmonologists on the 2009-2010 H1N1 Vaccination Effort
}

\author{
Sarah J. Clark, ${ }^{1}$ Anne E. Cowan, ${ }^{1}$ and Pascale M. Wortley ${ }^{2}$ \\ ${ }^{1}$ Child Health Evaluation and Research (CHEAR) Unit, University of Michigan, 300 N Ingalls, Room 6E06, Ann Arbor, \\ MI 48109-5456, USA \\ ${ }^{2}$ Immunization Services Division, NCIRD, Centers for Disease Control and Prevention, Atlanta, GA 30333, USA \\ Correspondence should be addressed to Sarah J. Clark, saclark@med.umich.edu
}

Received 27 September 2011; Accepted 9 November 2011

Academic Editor: Charlie Strange

Copyright (๑) 2012 Sarah J. Clark et al. This is an open access article distributed under the Creative Commons Attribution License, which permits unrestricted use, distribution, and reproduction in any medium, provided the original work is properly cited.

Persons with high-risk conditions such as asthma were a target group for H1N1 vaccine recommendations. We conducted a mailed survey of a national sample of pulmonologists to understand their participation in the 2009-2010 H1N1 vaccine campaign. The response rate was 59\%. The majority of pulmonologists strongly recommended H1N1 vaccine for children (73\%) and adults aged 25-64 years (51\%). Only 60\% of respondents administered H1N1 vaccine in their practice compared to $87 \%$ who offered seasonal influenza vaccine. Other than vaccine supply, respondents who provided H1N1 vaccine reported few logistical problems. Twothirds of respondents would be very likely to vaccinate during a future influenza pandemic; this rate was higher among those who provided $\mathrm{H} 1 \mathrm{~N} 1$ vaccine and/or seasonal flu vaccine. In total, the $\mathrm{H} 1 \mathrm{~N} 1$ vaccine-related experiences of pulmonologists seemed to be positive. However, additional efforts are needed to increase participation in future pandemic vaccination campaigns.

\section{Introduction}

In June 2009, the World Health Organization declared a worldwide pandemic of H1N1 influenza [1], and, in September 2009, a novel H1N1 influenza vaccine was licensed for use in the United States [2]. As with seasonal influenza, persons with asthma and other chronic respiratory conditions were considered at high risk for H1N1 influenza and its complications [3].

Early reports indicated high rates of asthma among those hospitalized and a younger age profile among those hospitalized than what is common for seasonal influenza [46]. These data underscored the importance of vaccinating high-risk children and nonelderly adults, as well as the need for effective strategies to reach these populations. In this context, pulmonologists represented a provider group that could vaccinate children and adults with asthma and other high-risk respiratory conditions.

Unlike a typical influenza season, in which physicians who offer flu vaccine purchase it in the private market, the federal government purchased $\mathrm{H} 1 \mathrm{~N} 1$ vaccine and gave state and local public health officials the responsibility for coordinating the $\mathrm{H} 1 \mathrm{~N} 1$ vaccination campaign in their jurisdiction.
These responsibilities included enrollment of providers as $\mathrm{H} 1 \mathrm{~N} 1$ vaccinators and distribution of $\mathrm{H} 1 \mathrm{~N} 1$ to enrolled providers. The extent to which state and local public health officials were able to involve subspecialists in the H1N1 vaccination campaign is unknown.

The goal of this study was to describe the experiences of pulmonologists related to the 2009-2010 H1N1 vaccination campaign to help inform future influenza vaccination campaigns.

\section{Methods}

2.1. Sample. Through a contracted vendor, we drew a national random sample of 1,998 pulmonologists from the American Medical Association (AMA) Physician Masterfile. The AMA Physician Masterfile is a comprehensive database of physicians licensed to practice in the United States, and includes both AMA members and nonmembers. Our sampling frame included all allopathic and osteopathic physicians in office- or hospital-based, direct patient care, with a self-reported primary specialty of pulmonary disease. Excluded were physicians $\geq 70$ years, in residency training or employed at federal medical facilities. 
2.2. Survey Design. The 4-page, 18-item survey instrument incorporated factors known to be related to influenza vaccination in general (e.g., strength of physician recommendation for vaccination [4-10], safety concerns about influenza vaccine $[4,5,8])$, as well as practical and logistical issues specific to the H1N1 vaccine campaign. Specific questions included the following:

(i) typical recommendation regarding $\mathrm{H} 1 \mathrm{~N} 1$ vaccination for patients by age group (strongly recommended, recommended, neutral, recommended against) and whether recommended $\mathrm{H} 1 \mathrm{~N} 1$ vaccine for patients during inpatient stays,

(ii) own and perceived patient level of concern with safety of $\mathrm{H} 1 \mathrm{~N} 1$ vaccine relative to that of seasonal flu vaccine (much less, somewhat less, about the same, somewhat greater, much greater),

(iii) level of participation in the 2009-2010 H1N1 vaccine campaign (agreed to provide H1N1 vaccine and received vaccine, agreed to provide $H 1 N 1$ vaccine but did not receive vaccine, did not agree to provide $H 1 N 1$ vaccine, and did not know about opportunities to provide H1N1 vaccine),

(iv) among $\mathrm{H} 1 \mathrm{~N} 1$ vaccinators, experiences with administering $\mathrm{H} 1 \mathrm{~N} 1$ vaccine, including month vaccine first available for patients, whether prioritized among adult patients when supply was limited and, if so, to which subgroups, and extent to which certain practical and logistical issues related to $\mathrm{H} 1 \mathrm{~N} 1$ vaccination were problematic (not a problem, minor problem, major problem),

(v) whether practice offered seasonal flu vaccine in 20092010 and planned to offer flu vaccine for the 20102011 influenza season,

(vi) likelihood of practice providing vaccine in the event of a future influenza pandemic (very likely, somewhat likely, neutral, somewhat unlikely, very unlikely), and

(vii) practice characteristics, including practice ownership/affiliation and whether provide inpatient care.

The University of Michigan Medical School Institutional Review Board approved this study.

2.3. Survey Administration. The initial survey mailing was sent in June 2010 and included a \$5 cash incentive. Two additional mailings to nonrespondents occurred at approximately 4-week intervals.

2.4. Data Analysis. We generated univariate frequencies for each variable and then performed chi-square analyses to examine associations between key survey variables (e.g., H1N1 vaccine recommendation, $\mathrm{H} 1 \mathrm{~N} 1$ vaccinator status, likelihood of participating in future influenza pandemic) and practice and personal demographic characteristics. A twotailed $\alpha$-level of 0.05 was the threshold for statistical significance. All analyses were conducted using SAS version 9.2 (SAS Institute, Cary, NC, USA).
TABLE 1: Respondent characteristics $(N=945)$.

\begin{tabular}{lc}
\hline & $\%$ \\
\hline Practice setting/affiliation & \\
$\quad$ Private independent practice/network, IPA & $82 \%$ \\
Hospital, Health system, Univ. medical center & $17 \%$ \\
$\quad$ Public clinic, Community health center, other & $1 \%$ \\
\hline Patient mix & \\
$\quad$ Pediatric and adult patients & $63 \%$ \\
$\quad$ Adult patients only & $37 \%$ \\
\hline Provide any inpatient care & \\
$\quad$ Yes & $91 \%$ \\
$\quad$ No & $9 \%$ \\
\hline Offered seasonal flu vaccine during the 2009-2010 \\
influenza season \\
$\quad$ Yes \\
$\quad$ No/Unsure \\
\hline
\end{tabular}

\section{Results}

3.1. Response Rate. Of the 1,998 pulmonologists in the mailing sample, 107 were excluded because mailing materials were returned as undeliverable. Completed surveys were returned by 1,114 pulmonologists, yielding a response rate of $59 \%$. Of these, 169 were ineligible because they do not provide any direct patient care in an outpatient setting. Thus, the final analytic sample included 945 respondents. Practice characteristics of respondents are presented in Table 1.

3.2. H1N1 Vaccine Recommendation. Respondents' strength of recommendation for $\mathrm{H} 1 \mathrm{~N} 1$ vaccine varied by patient age group (Table 2), with a strong recommendation most common for pediatric patients. There were no consistent or notable significant differences in the strength of $\mathrm{H} 1 \mathrm{~N} 1$ vaccine recommendation by practice characteristics for any of the three patient age groups.

3.3. H1N1 Vaccinator Status. As shown in Table 3, the majority $(60 \%)$ of respondents received $\mathrm{H} 1 \mathrm{~N} 1$ vaccine to administer to patients (i.e., were $\mathrm{H} 1 \mathrm{~N} 1$ vaccinators), while a small proportion agreed to participate but never received vaccine. Nearly one-third of respondents did not agree to participate or had not heard of opportunities to participate in the $\mathrm{H} 1 \mathrm{~N} 1$ vaccine campaign. $\mathrm{H} 1 \mathrm{~N} 1$ vaccinator status was associated with practice setting; all respondents in public clinics and three-quarters of those based in hospitals were $\mathrm{H} 1 \mathrm{~N} 1$ vaccinators. However, in private practice, the most common practice setting, just over half of respondents were H1N1 vaccinators. Pulmonologists whose practices offered seasonal flu vaccine in 2009-2010 were much more likely to be $\mathrm{H} 1 \mathrm{~N} 1$ vaccinators; less than $10 \%$ of those who did not offer seasonal flu vaccine were $\mathrm{H} 1 \mathrm{~N} 1$ vaccinators.

3.4. Vaccine Safety Concerns. Eleven percent of respondents reported that they had greater concerns about the safety 
TABLe 2: Pulmonologists' typical H1N1 vaccine recommendation by patient age group $(N=945)^{*}$.

\begin{tabular}{lcccc}
\hline For patients & Strongly recommended & Recommended & Neutral & $\begin{array}{c}\text { Recommended } \\
\text { against }\end{array}$ \\
\hline$\leq 18$ Years & $73 \%$ & $18 \%$ & $7 \%$ & $2 \%$ \\
$25-64$ Years & $51 \%$ & $39 \%$ & $9 \%$ & $1 \%$ \\
$\geq 65$ Years & $43 \%$ & $33 \%$ & $18 \%$ & $6 \%$ \\
\hline
\end{tabular}

${ }^{*}$ Results for each age group exclude respondents who do not provide care for patients of that age.

TABLE 3: H1N1 vaccinator status of pulmonologists and association with practice characteristics.

\begin{tabular}{|c|c|c|c|c|}
\hline Variable & $\begin{array}{l}\text { Agreed to } \\
\text { participate, } \\
\text { received vaccine }\end{array}$ & $\begin{array}{l}\text { Agreed to } \\
\text { participate, did } \\
\text { not receive } \\
\text { vaccine }\end{array}$ & $\begin{array}{l}\text { Did not agree to } \\
\text { participate }\end{array}$ & $\begin{array}{c}\text { Did not know about } \\
\text { opportunities to } \\
\text { participate }\end{array}$ \\
\hline Overall $(N=945)$ & $60 \%$ & $9 \%$ & $20 \%$ & $11 \%$ \\
\hline \multicolumn{5}{|l|}{ Practice setting/affiliation $* \dagger$} \\
\hline Private independent practice/network $(N=760)$ & $56 \%$ & $10 \%$ & $23 \%$ & $11 \%$ \\
\hline Hospital/health system $(N=165)$ & $77 \%$ & $5 \%$ & $9 \%$ & $9 \%$ \\
\hline Public clinic/CHC/other $(N=6)$ & $100 \%$ & $0 \%$ & $0 \%$ & $0 \%$ \\
\hline \multirow{2}{*}{\multicolumn{5}{|c|}{$\begin{array}{l}\text { Offered seasonal flu vaccine for } 2009-2010 \\
\text { influenza season* } * \dagger\end{array}$}} \\
\hline & & & & \\
\hline Yes $(N=805)$ & $68 \%$ & $10 \%$ & $14 \%$ & $8 \%$ \\
\hline No $(N=122)$ & $9 \%$ & $2 \%$ & $59 \%$ & $30 \%$ \\
\hline
\end{tabular}

of H1N1 vaccine than seasonal influenza vaccine, $59 \%$ had "about the same" level of concern, and 30\% had less concern about the safety of H1N1 vaccine. Respondents who had a greater concern about $\mathrm{H} 1 \mathrm{~N} 1$ vaccine safety were less likely to strongly recommend $\mathrm{H} 1 \mathrm{~N} 1$ vaccine than those whose level of concern was similar or lower; this finding was consistent for all three patient age groups $(P=.018$ for pediatric patients, and $P<.001$ for both adults 25-64 years and seniors). In addition, respondents who reported safety concerns were less likely to be $\mathrm{H} 1 \mathrm{~N} 1$ vaccinators compared to those with a similar or lower level of concern $(36 \%$ versus $63 \%, P<.001)$.

With regard to patients' safety concerns, $52 \%$ of respondents perceived that patients were more concerned with the safety of $\mathrm{H} 1 \mathrm{~N} 1$ vaccine relative to seasonal influenza vaccine. Another 33\% felt that their patients' level of concern was "about the same," while 15\% perceived patient concerns to be less for $\mathrm{H} 1 \mathrm{~N} 1$ vaccine than seasonal flu vaccine. Perceived patient concern was not associated with physicians' selfreported strength of recommendation for $\mathrm{H} 1 \mathrm{~N} 1$ vaccine or $\mathrm{H} 1 \mathrm{~N} 1$ vaccinator status.

3.5. Experiences of H1N1 Vaccinators. Among H1N1 vaccinators, the first month in which respondents reported having vaccine available for patients was 20\% October, $34 \%$ November, 20\% December, and 7\% January or later; 19\% were unsure of the timing of vaccine availability. Most H1N1 vaccinators offered vaccine at scheduled visits $(74 \%)$ or on a walk-in basis $(62 \%)$, with just under half (45\%) offering both options.
Based on the practical and logistical issues with H1N1 vaccination listed in Table 4, vaccine supply was the main challenge for $\mathrm{H} 1 \mathrm{~N} 1$ vaccinators. Across all of the issues listed in Table 4, 39\% of respondents rated one of these issues as a major problem, 13\% said two of these issues were major problems, $7 \%$ rated three or more as major problems, and $41 \%$ of respondents reported no major problems.

3.6. Future Participation in Influenza Vaccination. Most respondents $(86 \%)$ reported that their practice planned to offer seasonal flu vaccine for the 2010-2011 influenza season, which was similar to the proportion offering seasonal flu vaccine for the 2009-2010 season (87\%). A smaller proportion $(65 \%)$ said their practice would be "very likely" to provide vaccine in the event of a future influenza pandemic, with another 13\% indicating their practice would be "somewhat likely" to participate. An additional 8\% were neutral, 5\% said "somewhat unlikely," and 9\% said "very unlikely."

Being "very likely" to provide vaccine in a future influenza pandemic was more common among those who offered versus did not offer seasonal flu vaccine during the 20092010 influenza season (72\% versus $19 \%, P<.001)$ and among $\mathrm{H} 1 \mathrm{~N} 1$ vaccinators versus nonvaccinators ( $84 \%$ versus $34 \%, P<.001)$. Among $\mathrm{H} 1 \mathrm{~N} 1$ vaccinators, being "very likely" to participate in a future influenza pandemic was less common among those who rated two or more of the issues in Table 4 as major problems compared to those who said none or one of the issues was a major problem (64\% versus $86 \%$, $P=.002)$. 
TABLE 4: Extent of problems experienced by pulmonologists providing H1N1 vaccine $(N=564)$.

\begin{tabular}{|c|c|c|c|}
\hline & Not a problem & Minor problem & Major problem \\
\hline Inadequate or inconsistent $\mathrm{H} 1 \mathrm{~N} 1$ vaccine supply & $18 \%$ & $37 \%$ & $45 \%$ \\
\hline Lack of patient acceptance (demand) for $\mathrm{H} 1 \mathrm{~N} 1$ vaccine & $50 \%$ & $42 \%$ & $8 \%$ \\
\hline Elderly patients frustrated with lower priority for vaccine & $39 \%$ & $48 \%$ & $13 \%$ \\
\hline Staff unfamiliar with different $\mathrm{H} 1 \mathrm{~N} 1$ vaccine products & $78 \%$ & $21 \%$ & $1 \%$ \\
\hline Storage/handling of $\mathrm{H} 1 \mathrm{~N} 1$ vaccine & $83 \%$ & $15 \%$ & $2 \%$ \\
\hline Reimbursement/billing for vaccine administration & $70 \%$ & $24 \%$ & $6 \%$ \\
\hline $\mathrm{H} 1 \mathrm{~N} 1$ reporting requirements & $51 \%$ & $39 \%$ & $10 \%$ \\
\hline Return/disposal of unused H1N1 vaccine & $66 \%$ & $27 \%$ & $7 \%$ \\
\hline
\end{tabular}

\section{Discussion}

Summary data on hospitalizations and deaths resulting from the 2009-2010 H1N1 influenza [11, 12] confirmed initial reports [4-6] that persons with asthma and other chronic conditions were at higher risk for morbidity and mortality and, therefore, an important target for H1N1 vaccination. One strategy for reaching these populations was to include subspecialists, such as pulmonologists, in the 2009-2010 $\mathrm{H} 1 \mathrm{~N} 1$ vaccination campaign.

Assessing physician participation in public health campaigns is a critical aspect of understanding the extent to which a shared public health-medical delivery system is feasible for future pandemic-type events. A positive finding from this study is that a majority of pulmonologists surveyed participated in the 2009-2010 H1N1 vaccination campaign as $\mathrm{H} 1 \mathrm{~N} 1$ vaccinators. The importance of including physicians that can reach high-risk populations is supported by recent data showing that persons at high risk are more likely than those not at high risk to receive flu vaccine at a doctor's office versus other locations [13].

Of concern, however, is that participation was lowest in private pulmonology practices (respondents' most common practice setting) relative to hospital-based and public sector practices. This highlights a challenge for state and local public health officials in recruiting physicians to assist with pandemic vaccination campaigns. While these officials may be able to build upon existing relationships with hospitals to reach hospital-based providers, recruiting private practices is likely more time and resource intensive, as each practice must be contacted individually. In addition, from the physicians' perspective, smaller independent practices may be less likely to want, or to have the capacity, to take on an additional vaccine.

Participation in the H1N1 vaccination campaign among those who did not provide seasonal flu vaccine in 20092010 was much lower, and approximately one-third of these respondents said they did not know about opportunities to provide $\mathrm{H} 1 \mathrm{~N} 1$ vaccine, suggesting that it is particularly difficult to reach those who are not already "plugged in" to the immunization delivery system. For this reason, public health officials may find it easier to recruit primary care physicians for pandemic vaccination efforts. However, including subspecialists is important for reaching persons at high risk. For example, although the majority of ambulatory care visits for asthma and other chronic pulmonary conditions are to primary care offices, about one-third of these visits are to medical subspecialty offices [14]. Focusing solely on primary care physicians would likely miss a subset of this high-risk population.

Overall, a high proportion of pulmonologists recommended $\mathrm{H} 1 \mathrm{~N} 1$ vaccine to their patients. Given that virtually all of their patients less than 65 years of age would be classified as high risk for $\mathrm{H} 1 \mathrm{~N} 1$ vaccine, it is unclear why the proportion who "strongly recommended" vaccination was much higher for patients 18 years and younger compared to those aged 25-64 years. Because all children were an initial target group for vaccination, not just the high-risk subgroup, it may have been easier for physicians to emphasize vaccination for this patient group. It is also possible that state or local public health or medical community messages may have focused more attention on vaccinating children.

The proportion of pulmonologists who strongly recommended H1N1 vaccine to adults aged 25-64 years was only slightly higher than the proportion for adults aged 65 years and older, despite the fact that the latter population was not an initial target group for $\mathrm{H} 1 \mathrm{~N} 1$ vaccination. Expansion beyond the initial target groups was recommended first for all adults aged 25-64 years and then, once demand for the younger age groups was met, to those aged 65 years and over, regardless of high-risk status [3]. It is possible that some pulmonologists may have been unaware of the differential recommendations for these populations, or that, by the time vaccine was available in their practice setting, the target group recommendations were no longer needed. It is also possible that some pulmonologists disagreed with the recommendations, feeling that the presence of a high-risk pulmonary condition preempted an age-based determination.

While the proportion of respondents having greater concerns with the safety of $\mathrm{H} 1 \mathrm{~N} 1$ vaccine relative to seasonal flu vaccine was not large, those with greater concerns were much less likely to strongly recommend $\mathrm{H} 1 \mathrm{~N} 1$ vaccine to their patients and participate as $\mathrm{H} 1 \mathrm{~N} 1$ vaccinators. Future campaigns should stress the importance of proactive and widespread messages directed toward physicians regarding vaccine safety.

By far the biggest challenge faced by $\mathrm{H} 1 \mathrm{~N} 1$ vaccinators was the limited supply of vaccine. Although the majority of respondents reported receiving vaccine by November, the peak of disease, and likely of patient demand, occurred prior 
to widespread vaccine availability. This was a significant and broadly experienced challenge during the $\mathrm{H} 1 \mathrm{~N} 1$ vaccination campaign [15] and also varied by state or local area. Otherwise, few issues caused major problems for pulmonologists providing H1N1 vaccine, and many reported experiencing no major problems.

A majority of respondents felt their practice would be "very likely" to provide vaccine in the event of a future influenza pandemic. Respondents who were $\mathrm{H} 1 \mathrm{~N} 1$ vaccinators were much more likely to express willingness to participate in the event of a future pandemic, as were those who provided seasonal flu vaccine in 2009-2010. Although the proportion very likely to participate in a future influenza pandemic was similar to the proportion that was $\mathrm{H} 1 \mathrm{~N} 1$ vaccinators, it is markedly lower than the proportion providing seasonal flu vaccine, implying that outpatient pulmonologists do not see themselves as essential to a pandemic response. Public health and emergency preparedness officials may want to work toward including subspecialty physicians, such as pulmonologists, in short-term planning efforts, in order to accomplish longer-term objective of creating a broad base of vaccinators for pandemic response. In addition, pulmonologists interested in vaccinating can be proactive in reaching out to public health officials during a pandemic. Strategies for encouraging pulmonologists' participation in pandemic vaccination should continue to be explored.

\section{Limitations}

As with any survey study, there are limitations of response bias; however, the response rates obtained in this study were quite favorable, compared to other physician surveys. Also, given the sample size, we were unable to make statistically viable state-by-state comparisons of the results. The major limitation with this study is that our pulmonologist sample is not generalizable to the full universe of pulmonologists. We included physicians in our sample whose self-reported primary specialty is pulmonary disease but did not include the other potentially applicable primary specialty of pulmonary critical care medicine. To be eligible for our study, respondents needed to provide patient care in an outpatient setting, and we felt that a high proportion of physicians reporting pulmonary critical care medicine as their primary specialty would not provide outpatient care. As it turns out, respondents reporting a specialty of pulmonary disease are skewed to older, male physicians; the sample included no one under the age of 40 years. In exploring this issue further, we found that recent research has shown that pulmonology alone is not a popular choice among internal medicine residents and that pulmonology with critical care is more likely to be chosen than either pulmonology or critical care alone [16]. We went back to the contracted vendor and requested year of birth frequency counts from the AMA Masterfile, using our initial sample specifications, for all physicians reporting a primary specialty of either pulmonary critical care medicine or pulmonary disease. Comparing year of birth data across the two groups confirmed that younger physicians are reporting pulmonary critical care medicine as their primary specialty to the exclusion of pulmonary disease. Given this limitation, our results likely under-report the experiences of hospital-based pulmonologists and likely provide a conservative estimate of pulmonologists' participation in the $\mathrm{H} 1 \mathrm{~N} 1$ vaccination campaign.

\section{Conclusion}

Federal, state, and local public health officials should be encouraged to include pulmonologists in future pandemic influenza planning and vaccination efforts as an effective strategy for reaching persons with asthma and other chronic respiratory conditions. The experiences of a subset of pulmonologists in the H1N1 vaccine campaign were generally positive, with the majority recommending the vaccine to their patients and serving as H1N1 vaccinators. However, there is much room for improvement in terms of participation levels, and the challenges related to participation by private practices must be further explored.

\section{Acknowledgments}

This study was funded by the Centers for Disease Control and Prevention, through a cooperative agreement with the Association of Prevention Teaching and Research. The findings are those of the authors and do not represent an official viewpoint of the CDC.

\section{References}

[1] M. Chan, World now at the start of 2009 influenza pandemic. Statement to the press by WHO Director-General Dr. Margaret Chan, June 2009, http://www.who.int/mediacentre/ news/statements/2009/h1n1_pandemic_phase6_20090611/en/ index.html.

[2] US Food and Drug Administration. FDA Approves Vaccines for 2009 H1N1 Influenza Virus. Press release, September 2009, http://www.fda.gov/newsevents/newsroom/pressannouncements/ucm182399.htm.

[3] Centers for Disease Control and Prevention, "Use of influenza A (H1N1) 2009 monovalent vaccine: recommendations of the Advisory Committee on Immunization Practices (ACIP), 2009," Morbidity and Mortality Weekly Report, vol. 58, no. RR10 , pp. 1-8, 2009.

[4] S. Jain, L. Kamimoto, A. M. Bramley et al., "Hospitalized patients with $2009 \mathrm{H} 1 \mathrm{~N} 1$ influenza in the United States, AprilJune 2009," New England Journal of Medicine, vol. 361, no. 20, pp. 1935-1944, 2009.

[5] Centers for Disease Control and Prevention, "Hospitalized patients with novel influenza A (H1N1) virus infection-California, April-May, 2009," Morbidity and Mortality Weekly Report, vol. 58, no. 19, pp. 536-541, 2009.

[6] J. K. Louie, M. Acosta, K. Winter et al., "Factors associated with death or hospitalization due to pandemic 2009 influenza $\mathrm{A}(\mathrm{H} 1 \mathrm{~N} 1)$ infection in California," Journal of the American Medical Association, vol. 302, no. 17, pp. 1896-1902, 2009.

[7] M. H. Rathore, A. Mirza, A. Subedar et al., "Influenza vaccine: awareness and barriers to immunization in families of children with chronic medical conditions other than asthma," Southern Medical Journal, vol. 101, no. 11, pp. 1101-1105, 2008.

[8] S. K. Gnanasekaran, J. A. Finkelstein, K. Hohman, M. O’Brien, B. Kruskal, and T. A. Lieu, "Parental perspectives on influenza 
vaccination among children with asthma," Public Health Reports, vol. 121, no. 2, pp. 181-188, 2006.

[9] M. F. Daley, L. A. Crane, V. Chandramouli et al., "Influenza among healthy young children: changes in parental attitudes and predictors of immunization during the 2003 to 2004 influenza season," Pediatrics, vol. 117, no. 2, pp. e268-e277, 2006.

[10] R. C. Chi and K. M. Neuzil, "The association of sociodemographic factors and patient attitudes on influenza vaccination rates in older persons," American Journal of the Medical Sciences, vol. 327, no. 3, pp. 113-117, 2004.

[11] J. Skarbinski, S. Jain, A. Bramley et al., "Hospitalized patients with 2009 pandemic influenza a (H1N1) virus infection in the United States-September-October 2009," Clinical Infectious Diseases, vol. 52, supplement 1, pp. S50-S59, 2011.

[12] A. L. Fowlkes, P. Arguin, M. S. Biggerstaff et al., "Epidemiology of 2009 pandemic influenza a $(\mathrm{H} 1 \mathrm{~N} 1)$ deaths in the United States, April-July 2009," Clinical Infectious Diseases, vol. 52, supplement 1, pp. S60-S68, 2011.

[13] E. D. Kennedy, T. A. Santibanez, L. N. Bryan et al., "Place of influenza vaccination among adults-United States, 2010-11 influenza season," Morbidity and Mortality Weekly Report, vol. 60, no. 23, pp. 781-785, 2011.

[14] S. M. Schappert and E. A. Rechtsteiner, "Ambulatory medical care utilization estimates for 2007," Vital and Health Statistics. Series 13, Data from the National Health Survey, no. 169, pp. 1-38, 2011.

[15] Institute of Medicine (IOM), The 2009 H1N1 Influenza Vaccination Campaign: Summary of a Workshop Series, The National Academies Press, Washington, DC, USA, 2010.

[16] S. Lorin, J. Heffner, and S. Carson, "Attitudes and perceptions of internal medicine residents regarding pulmonary and critical care subspecialty training," Chest, vol. 127 , no. 2, pp. 630 636, 2005. 


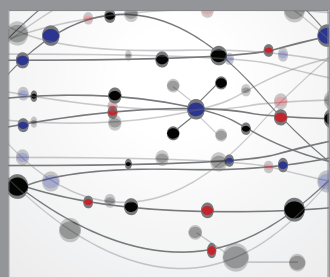

The Scientific World Journal
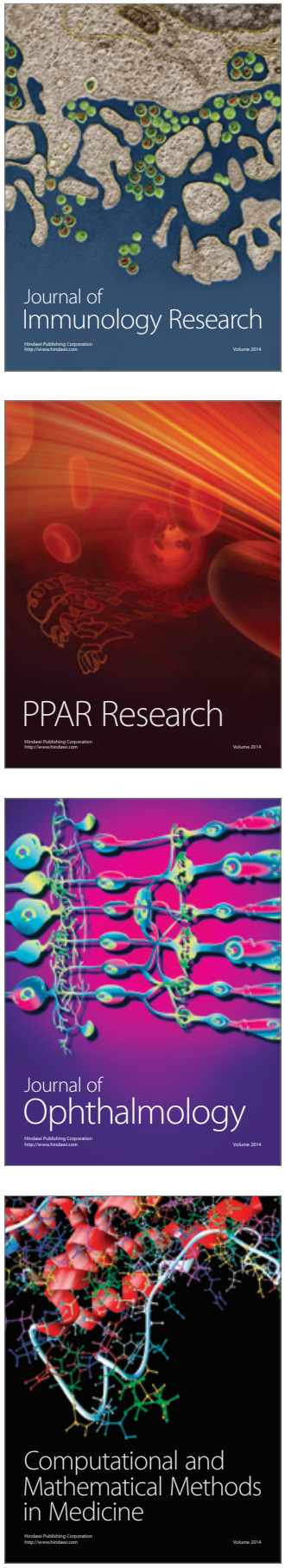

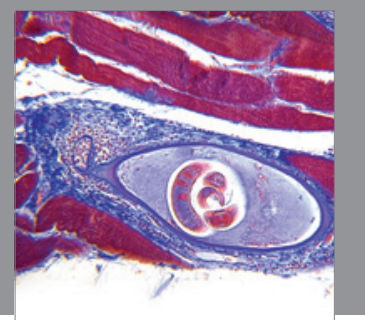

Gastroenterology

Research and Practice
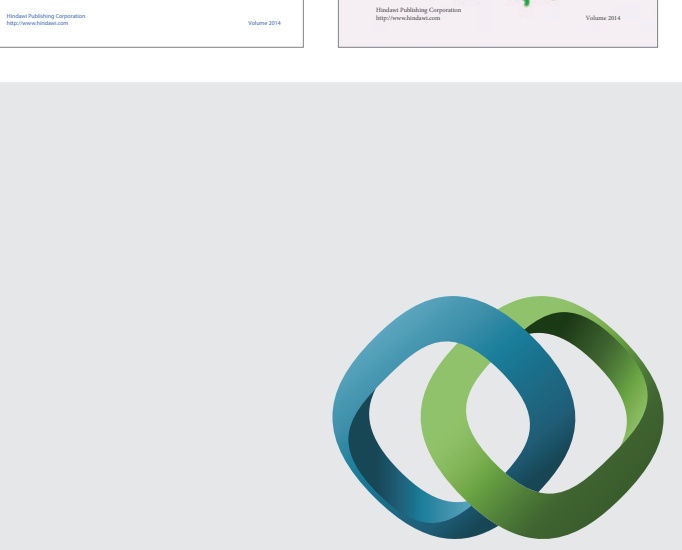

\section{Hindawi}

Submit your manuscripts at

http://www.hindawi.com
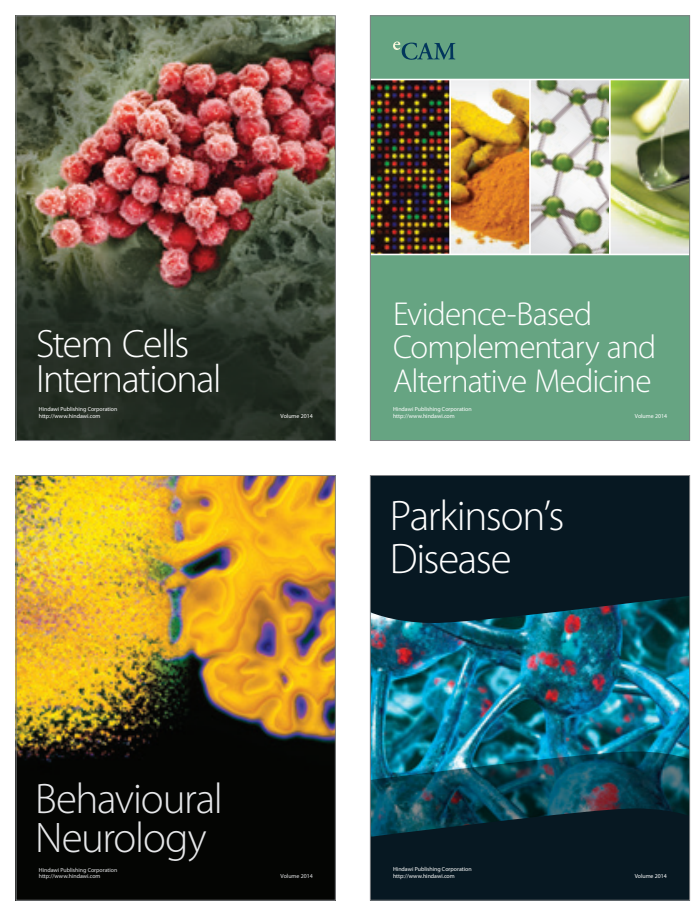

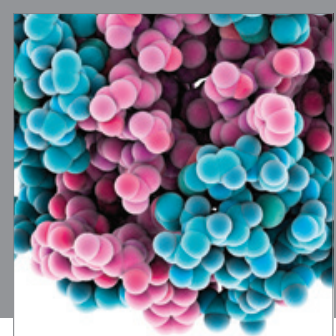

Journal of
Diabetes Research

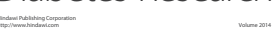

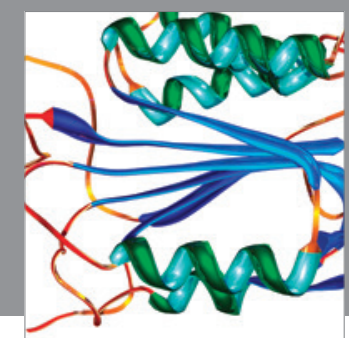

Disease Markers
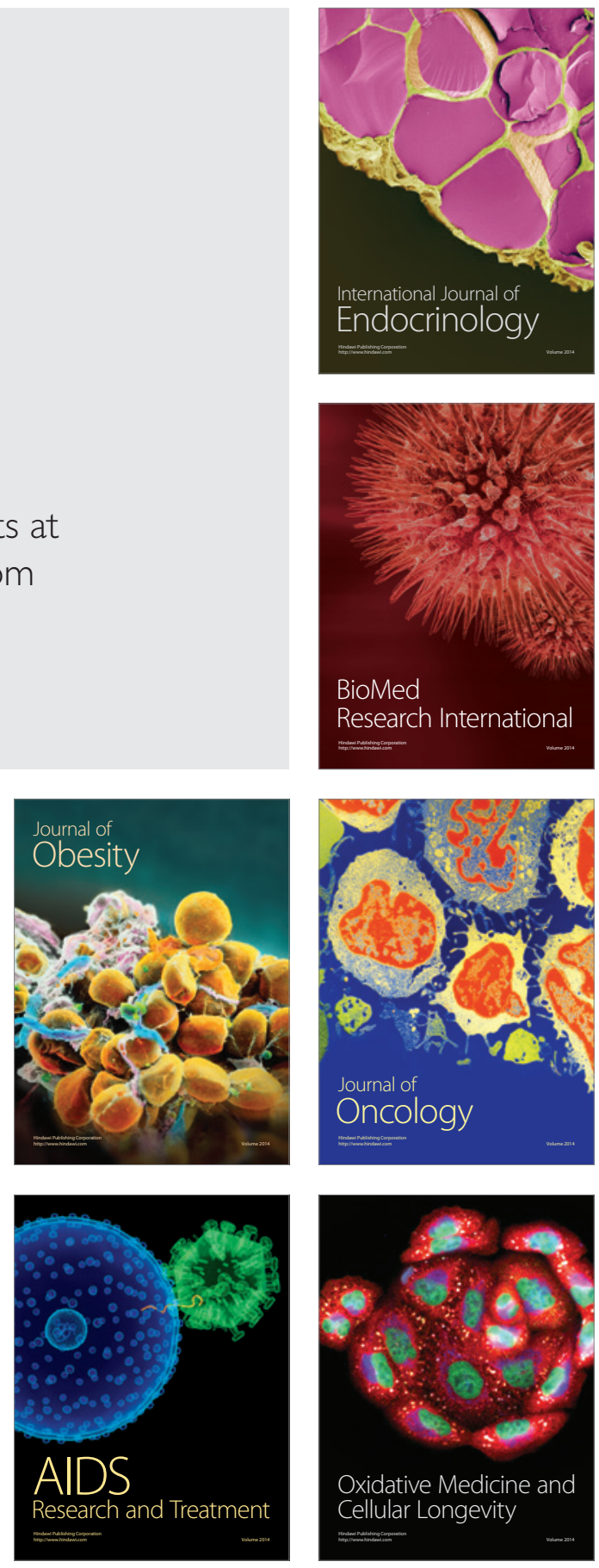